\title{
Critical care for lung cancer patients: surgical treatment during COVID-19 pandemic
}

\author{
Gokce $\mathrm{A}^{1}$, Hatipoglu $\mathrm{M}^{1}$, Akboga $\mathrm{SA}^{1}$, Sezen $\mathrm{AI}^{2}$, Akkas $\mathrm{Y}^{1}$, Kocer $\mathrm{B}^{1}$ \\ University of Health Sciences, Ankara City Hospital, Department of Thoracic Surgery, \\ Bilkent, Ankara, Turkey. anil66gokce@hotmail.com
}

\section{ABSTRACT}

BACKGROUND: During the COVID-19 pandemic, some factors have led to changes in the management of patients with lung cancer. In our study, we aimed to present our surgical treatment approach to patients with NSCLC during the COVID-19 pandemic.

METHODS: Patients who underwent surgery for NSCLC in our thoracic surgery clinic between March 2020 and March 2021 were evaluated retrospectively. The patients operated on were retrospectively evaluated in terms of sex, age, tumor staging, lung resection type, histopathological type, COVID-19 status, length of stay, complications, and mortality.

RESULTS: Thirty-five patients, 27 men and 8 women, underwent surgery for lung cancer. The 2 most common types of surgery were lobectomy (in 32 patients) and pneumonectomy (in 3 patients). According to cancer staging based on 8th TNM, 14 patients were stage 2B, 12 patients were stage $2 \mathrm{~A}$, and 9 patients were stage $3 \mathrm{~A}$. The morbidity rate was $14 \%$. No postoperative mortality was observed. Nine patients had a history of COVID- 19 before surgery. No significant difference was found in terms of complications in patients with a preoperative history of COVID-19. In the postoperative period, COVID-19 was observed in no patient in our clinic.

CONCLUSION: We think that surgical treatments should not be postponed for diseases such as lung cancer where the mortality rate is high and early diagnosis and treatment are very important. There will be no delay or inadequacy in the treatment of patients if the rules determined during the COVID-19 pandemic and other types of pandemic possibly occurring in the future are followed (Tab. 1, Ref. 23). Text in PDF www.elis.sk KEY WORDS: COVID-19, pandemic, lung cancer, surgical treatment, thoracic surgery.

\section{Introduction}

COVID-19 has caused a large number of deaths and infections worldwide since it started to spread in late 2019. Many medical centers aim to control its spread by implementing isolation protocols (1-3). While non-small cell lung cancer (NSCLC) remains a major cause of death, many elective procedures and operations for the treatment of diseases have been delayed (4-9). During the COVID-19 pandemic, some factors have led to changes in the management of patients with lung cancer. Decreased availability of hospital beds and intensive care beds in particular has caused delays in medical and surgical care for patients with lung cancer (10). Lung cancer screening programs have been temporarily interrupted and patients have had concerns about coming to the clinic $(11,12)$.

${ }^{1}$ University of Health Sciences, Ankara City Hospital, Department of Thoracic Surgery, Bilkent, Ankara, Turkey, and ${ }^{2}$ Istanbul Bakirkoy Dr. Sadi Konuk, Education and Research Hospital, Department of Infectious Diseases, Istanbul, Turkey

Address for correspondence: A. Gokce, MD, Thoracic Surgeon, University of Health Sciences, Ankara, City Hospital, Department of Thoracic Surgery, Bilkent, Ankara, Turkey.

Phone: +90 3125526000
Some medical centers have reorganized their activities for the surgical treatment of NSCLC in emergencies due to the COVID-19 pandemic (13). Diagnostic and therapeutic methods have been improved. Effective and timely treatment of NSCLC was enabled. In our study, we aimed to present our treatment approach to patients with NSCLC during the COVID-19 pandemic.

\section{Materials and methods}

The approval for the study was obtained from the local ethics committee. Patients who underwent surgery for NSCLC in our thoracic surgery clinic between March 2020 and March 2021 were evaluated retrospectively. Thirty-five patients were included in the study. The patients operated on were retrospectively evaluated in terms of sex, age, COVID-19 status, length of stay, complications, and mortality. Thoracotomy and video-assisted thoracic surgery were performed in the patients. The patients were evaluated in terms of complications such as infection, bleeding, arrhythmia, embolism, wound infection, and pneumonia which are the most common complications in thoracic surgery. In addition, the methods applied in order to protect both the healthcare personnel and patients from COVID-19 during the pandemic were reviewed. In the preoperative period, examinations were performed in terms 
of surgical preparation and infection. Consultations were held regarding additional diseases. The COVID-19 PCR test was performed at least once before surgery. In the postoperative period, the follow-up and examinations were conducted for complications and COVID-19 infection. The patients were invited to attend a further check-up 10 days after their discharge. During this period, the patients were followed up.

\section{Results}

During the study period, in our hospital, nearly 30,000 COVID-19 patients were followed up in the wards and intensive care units. During the 1-year study period of pandemic in our clinic, 35 patients, 27 men and 8 women, underwent surgery for lung cancer (Tab. 1). Their mean age was 57.7 years. The 2 most common types of surgery were lobectomy (in 32 patients), and pneumonectomy (in 3 patients). According to cancer staging based on 8 th TNM, 14 patients were stage $2 \mathrm{~B}, 12$ patients were stage $2 \mathrm{~A}$, and 9 patients were stage $3 \mathrm{~A}$. The 3 most common symptoms were chest pain (in 14 patients), shortness of breath (in 4 patients), and cough (in 2 patients). Fifteen patients did not have any symptoms at the time of admission to our clinic. Postoperatively, wound infection was observed in 2 patients, arrhythmia in 1 patient, prolonged air leak in 1 patient, and bacterial pneumonia in 1 patient. The morbidity rate was $14 \%$. No

Tab. 1. Nearly 30,000 patients with COVID-19 were followed in wards and intensive care units.

\begin{tabular}{|c|c|}
\hline & $\begin{array}{l}\text { Study population } \\
\mathrm{n}=35\end{array}$ \\
\hline \multicolumn{2}{|l|}{ Gender } \\
\hline male & 27 \\
\hline female & 8 \\
\hline mean age & 57.7 \\
\hline Age range (years) & $43-76$ \\
\hline \multicolumn{2}{|l|}{ Symptoms } \\
\hline no symptoms & 15 \\
\hline chest pain & 14 \\
\hline cough & 2 \\
\hline shortness of breath & 4 \\
\hline \multicolumn{2}{|l|}{ Tumour stage (8 TNM) } \\
\hline stage $2 \mathrm{~A}$ & 12 \\
\hline stage $2 \mathrm{~B}$ & 14 \\
\hline stage $3 \mathrm{~A}$ & 9 \\
\hline Preoperative COVID-19 & 9 patients \\
\hline Postoperative COVID-19 & 0 patients \\
\hline The average number of PCR tests performed & 1.8 \\
\hline Mean length of stay (days) & 11.3 \\
\hline \multicolumn{2}{|l|}{ Operation types } \\
\hline lobectomy & 32 \\
\hline pneumonectomy & 3 \\
\hline \multicolumn{2}{|l|}{ Postoperative complications } \\
\hline no complications & 30 \\
\hline wound infections & 2 \\
\hline arrhythmia & 1 \\
\hline pneumonia & 1 \\
\hline prolonged air leak & 1 \\
\hline
\end{tabular}

postoperative mortality was observed. Appropriate antibiotic therapy and frequent dressing were applied for the most common wound infection. Respiratory physiotherapy and appropriate medical treatment were applied for the treatment of pneumonia. For the treatment of arrhythmia and embolism, the patients were referred to the relevant clinics and the recommended treatments were applied fully. No postoperative mortality was observed. The mean number of PCR tests performed in the preoperative period for COVID-19 disease was 1.8. Nine patients had a history of COVID-19 before surgery. No significant difference was found in terms of complications in patients with a preoperative history of COVID-19. In the postoperative period, COVID-19 was observed in no patient in our clinic. We presume that the effective measures implemented by the surgical team were responsible for COVID-19 disease not being seen in any patient in the postoperative period.

\section{Discussion}

Standard assessment for NSCLC includes perioperative evaluation for imaging studies, biopsy, and surgery. Staging with computed tomography (CT) and positron emission tomography (PET) is an acceptable assessment of nodal metastasis or distant metastatic disease. Bronchoscopy and endobronchial ultrasound (EBUS) are used in cases in which mediastinal lymph node metastasis is suspected due to enlarged lymph nodes (greater than 1 $\mathrm{cm}$ ) or hypermetabolic fluorodeoxyglucose F18. The COVID-19 virus enters the upper respiratory tract and can become aerosolized during bronchoscopy. Recent experimental studies of the virus have shown the presence of prolonged COVID-19 aerosols in the air for up to 3 hours (14). Considering the risk of hospitalacquired transmission of COVID-19 during aerosol-generating procedures, we tried to minimize the use of bronchoscopy and it was usually performed during resection. In patients with unknown or suspected COVID-19 status, cervical mediastinoscopy was used as an alternative to EBUS for surgical staging of the mediastinum to avoid bronchoscopy.

The evaluation of lung function is very important before surgical resection for lung cancer. Standard pulmonary function testing with spirometry and diffusing capacity (DLCO) is the most common check of lung reserve prior to lung resection. Forced expiratory volume (FEV-1) and DLCO in 1 second are routinely measured during pulmonary function testing. During the COVID-19 pandemic, the pulmonary function laboratory in our hospital was closed and the tests were postponed to minimize the risk of SARS-CoV-2 infection. Alternative methods were used to measure pulmonary reserve and determine pulmonary function during the initial surgical evaluation and determination of suitability for resection. The stair-climbing test is a potential alternative for assessing the functional level. Climbing two floors of stairs without desaturation on pulse oximetry is a good indication that the patient may tolerate lobectomy. Moreover, it was stated that the 6-minute walk test may be applied for patients and that it may provide pulse oximetry values that correlate well with FEV1 and DLCO in patients with chronic obstructive pul- 
monary disease $(15,16)$. In addition to all these methods in our clinic, arterial blood gas evaluation was used to determine respiratory function.

The increase in COVID-19 cases has put a significant pressure on hospital beds and ventilators in this country. Elective operative cases have been significantly limited or postponed altogether due to the incidence of COVID-19. Timely planning of lung cancer resections has been difficult given the limited operating room capacity during the COVID-19 pandemic. Surgical resection with lymph node dissection is still the gold standard for NSCLC treatment. The American College of Surgeons and similar associations have prepared guidelines for cancer surgery triage during COVID-19, originally published in late March 2020, outlining general principles for prioritizing surgical cases, including chest malignancies, in the acute stages of the pandemic (17). Although the doubling time of lung cancer cells is between 45 and 200 days, the tumor volume may grow rapidly (15). An analysis of the United States National Database showed that a delay in treatment of more than 8 weeks is an independent risk factor for disease progression in NSCLC (16). Therefore, the delays of more than 2 months may lead to progression of the disease, especially in patients awaiting surgery for early-stage lung cancer. Surgical treatment is also the gold standard in eligible patients with locally advanced lung cancer. However, because locally advanced patients generally are at advanced age and have additional diseases, it was thought that the surgical treatment to be applied during the pandemic may pose a high risk. For this reason, there are publications in the literature stating that non-surgical treatment should also be applied to these patients who are to undergo surgical treatment $(18,19)$. Surgical treatment was successfully applied to every patient who could be treated in our clinic, regardless of their stage.

During the COVID-19 pandemic, few findings have been reported on the safety of performing surgery. The data of 4 patients who became infected with COVID-19 showed a high mortality rate of $75 \%$ (20). Cai et al showed that 7 patients tested positive for COVID-19 with a $43 \%$ mortality rate after lung resection (21). Li et al stated that 25 patients had COVID-19 with a 20 $\%$ mortality rate after thoracic surgery (22). A publication from Italy evaluated the safety of surgery during COVID-19; 71 lung resections were performed without any reported postoperative COVID-19 (23). Our results show the feasibility and safety of performing surgical resection for lung cancer in a hospital with a high number of COVID-19 patients (80\% capacity). Resection was performed in 30 patients with NSCLC or high suspicion of lung cancer. No major postoperative complications or mortality were observed in the patients, and no patient was infected with COVID-19 postoperatively. All procedures were performed using a minimally invasive approach with a short median hospital stay. Factors that may have led to our results include our current safety procedure, availability of protective equipment at all times, $\mathrm{CO}$ VID-19 testing for all patients and healthcare personnel, daily fever and symptom checks for all operating room personnel to support PCR testing, use of N-95 masks by all personnel, limiting operating room personnel numbers during intubation, use of minimal invasive techniques, and postoperative management in special areas. For patient and healthcare staff safety, it was important to have the capacity to perform PCR testing on the day before or in the morning of the planned operation and test the entire hospital staff at various stages. We prioritized resections of patients at higher risk (solid nodule component $>2 \mathrm{~cm}$, SUVmax $\geq 2.5$, or change in short-range imaging) associated with higher malignancy rate and disease progression. The only exceptions for deferred cases were patients with low probability of metastasis, slow growing cancers, biopsy-proven carcinoid diagnosis, those who refused surgical resection, and 2 patients who tested positive for COVID-19. Other patients chose to be followed up with planned surgical resection.

\section{Conclusion}

During the COVID-19 pandemic, thoracic surgery takes the highest risks due to direct contact with the lungs, especially in terms of surgery and postoperative period. We think that these risks will be minimized and health professionals will work safely due to the measures implemented during all processes. Moreover, we think that surgical treatments should not be postponed for diseases such as lung cancer, where the mortality rate is high and early diagnosis and treatment are very important. There will be no delay or inadequacy in the treatment of patients if the rules determined during the COVID-19 pandemic and other types of pandemics occurring possibly in the future are followed.

\section{References}

1. Ciceri F, Castagna A, Rovere-Querini P, De Cobelli F, Ruggeri A, Galli L et al. Early predictors of clinical outcomes of COVID-19 outbreak in Milan, Italy. Clin Immunol 2020; 217: 108509.

2. Franchini S, Spessot M, Landoni G, Piani C, Cappelletti C, Mariani F et al. Stranger months: how SARS-CoV-2, fear of contagion, and lockdown measures impacted attendance and clinical activity during February and March 2020 at an Urban Emergency Department in Milan. Disaster Med Public Health Prep 2020; 1-10.

3. Verelst F, Kuylen E, Beutels P. Indications for healthcare surge capacity in European countries facing an exponential increase in coronavirus disease (COVID-19) cases, March 2020. Euro Surveill 2020; 25 (13): 2000323.

4. Bilkhu R, Billè A. Elective lung cancer surgery in the COVID-19 era: how do we do it? Tumori 2020; 106 (3): 190-192.

5. Dingemans AC, Soo RA, Jazieh AR, Rice SJ, Kim YT, Teo LLS et al. Treatment guidance for patients with lung cancer during the coronavirus 2019 pandemic. J Thorac Oncol 2020; 15 (7): 1119-1136.

6. Luo J, Rizvi H, Preeshagul IR, Egger JV, Hoyos D, Bandlamudi C et al. COVID-19 in patients with lung cancer. Ann Oncol 2020; 31 (10): 1386-1396.

7. Chouaid C. Cancer du poumon à l'ère du COVID-19. Rev Malad Respir Actual 2020; 12 (2): 2S378-382.

8. Russano M, Citarella F, Vincenzi B, Tonini G, Santini D. Coronavirus disease 2019 or lung cancer: what should we treat? J Thorac Oncol 2020; 15 (7): e105-106. 


\section{$125-128$}

9. Liang W, Guan W, Chen R, Wang W, Li J, Xu K et al. Cancer patients in SARS-CoV-2 infection: a nationwide analysis in China. Lancet Oncol 2020; 21 (3): 335-337.

10. Bakhribah H, Zeitouni M, Daghistani RA, Almaghraby HQ, Khankan AA, Alkattan KM et al. Implications of COVID-19 pandemic on lung cancer management: a multidisciplinary perspective. Crit Rev Oncol Hematol 2020; 156: 103120.

11. Depypere LP, Daddi N, Gooseman MR, Batirel HF, Brunelli A. The impact of coronavirus disease 2019 on the practice of thoracic oncology surgery: a survey of members of the European Society of Thoracic Surgeons (ESTS). Eur J Cardiothorac Surg 2020; 58 (4): 752-762,

12. Mejía LH, Román AR, Barturen MG, Pelaez MMC, Campo-Canaveral de la Cruz JL, Naranjo JM et al. Thoracic surgery during the coronavirus disease 2019 (COVID-19) pandemic in Madrid, Spain: singlecentre report. Eur J Cardiothorac Surg 2020; 58 (5): 991-996.

13. Zangrillo A, Beretta L, Silvani P, Colombo S, Scandroglio AM, Dell'Acqua A et al. Fast reshaping of intensive care unit facilities in a large metropolitan hospital in Milan, Italy: facing the COVID-19 pandemic emergency. Crit Care Resusc 2020; 22 (2): 91-94.

14. Van Doremalen N, Bushmaker T, Morris DH, Holbrook MG, Gamble A, Williamson BN et al. Aerosol and surface stability of SARS-CoV-2 as compared with SARS-CoV-1. N Engl J Med 2020; 382 (16): 1564-1567.

15. Agrawal MB, Awad NT. Correlation between six minute walk test and spirometry in chronic pulmonary disease. J Clin Diagn Res 2015; 9 (8): OC01-4.
16. Fujimoto H, Asai K, Watanabe T, Kanazawa H, Hirata K. Association of six-minute walk distance (6MWD) with resting pulmonary function in patients with chronic obstructive pulmonary disease (COPD). Osaka City Med J 2011; 57 (1): 21-29.

17. American College of Surgeons ACS guidelines for triage and management of elective cancer surgery cases during the acute and recovery phases of coronavirus disease 2019 (COVID-19).

18. Brindle M, Gawande A. Managing COVID-19 in surgical systems. Ann Surg 2020; 272 (1): e1-e2.

19. Murthy S, Gomersall CD, Fowler RA. Care for critically ill patients with COVID-19. JAMA 2020; 323 (15): 1499-1500.

20. Aminian A, Safari S, Razheghian-Jahromi A, Ghorbani M, Delaney CP. COVID-19 outbreak and surgical practice: unexpected fatality in perioperative period. Ann Surg 2020; 272 (1): e27-e29.

21. Cai Y, Hao Z, Gao Y, Ping W, Wang Q, Pend S et al. Coronavirus disease 2019 in the perioperative period of lung resection: a brief report from a single thoracic surgery department in Wuhan, People's Republic of China. J Thorac Oncol 2020; 15 (6): 1065-1072.

22. Li Y, Peng S, Li L, Wang Q, Ping W, Zhang $\mathbf{N}$ et al. Clinical and transmission characteristics of COVID-19-a retrospective study of 25 cases from a single thoracic surgery department. Curr Med Sci 2020: 16.

23. Maurizi G, Rendina EA. A high-volume thoracic surgery division into the storm of the COVID-19 pandemic. Ann Thorac Surg 2020; 110 (2): 353-354. 